\title{
Dimensionamento dos profissionais de enfermagem No pronto socorro: uma revisão narrativa
}

\section{Nurse staffing in the emergency department: a narrative review}

DOI: $10.54018 /$ shsv1n1-002

Recebimento dos originais: 06/01/2020

Aceitação para publicação: 31/01/2020

\author{
Rafael de Freitas \\ Enfermeiro, pela Faculdade Integrada de Santa Maria (FISMA), Santa Maria, RS, \\ Brasil. E-mail: rafaelfreitasa@gmail.com
}

\section{Fernanda Stock da Silva}

Enfermeira, Mestre em enfermagem pela Universidade Federal de Santa Maria, Santa Maria, RS, Brasil. E-mail: fernandastock@yahoo.com.br

\section{Caren Franciele Coelho Dias}

Enfermeira, Mestre em enfermagem pela Universidade Federal de Santa Maria, Santa Maria, RS, Brasil. E-mail: carenfrancielecoelhodias@yahoo.com.br

\section{Luis Felipe Machado da Silva}

Enfermeiro, pela Faculdade Integrada de Santa Maria (FISMA), Santa Maria, RS, Brasil. E-mail: philpi@hotmail.com

\section{RESUMO}

O dimensionamento de profissionais de enfermagem é uma dificuldade enfrentada pela classe atualmente, é parte do processo inicial do método utilizado para alocar determinado número de profissionais que tem como objetivo prever a quantidade de funcionários por categoria. Objetivou-se identificar o que se tem publicado na literatura nacional acerca do dimensionamento de pessoal de enfermagem no PS. Trata-se de uma revisão bibliográfica do tipo narrativa. A pesquisa foi realizada na Biblioteca Virtual em Saúde (BVS), nas bases de dados Literatura Latino Americana e do Caribe em Ciências da saúde (LILACS), a partir da estratégia de busca: enfermagem "and" urgência "and" emergência "and" dimensionamento, quatro artigos foram selecionados para o presente estudo. O levantamento de dados ocorreu no período de março de 2019. A partir da análise das produções, foram constituídas duas categorias temáticas: "Perfil da Enfermagem em Pronto Socorro, imaturidade profissional e inexperiência: uma realidade na assistência de enfermagem" e "Peculiaridade de um Pronto Socorro no Dimensionamento de Enfermagem". Os estudos apontaram que o dimensionamento de enfermagem é uma das fragilidades da gestão hospitalar e merece uma ênfase por parte dos gestores, a fim de evitar uma sobrecarga da equipe atuante do setor e comprometer a assistência de enfermagem ao paciente. O dimensionamento de enfermagem é um dos problemas mais difíceis e complexos nas instituições 
hospitalares, mas merece uma atenção especial por parte dos gestores visto que a unidade de PS oferece variáveis que influem no processo de dimensionamento.

Palavras-chave: Enfermagem; Serviço hospitalar de emergência; Administração de recursos humanos em hospitais.

\section{ABSTRACT}

The dimensioning of nursing professionals is a difficulty faced by the class today, it is part of the initial process of the method used to allocate a certain number of professionals, which aims to predict the number of employees per category. The objective was to identify what has been published in the national literature about the dimensioning of nursing staff in the ER. This is a literature review of the narrative type. The research was carried out in the Virtual Health Library (VHL), in the Latin American and Caribbean Literature in Health Sciences (LILACS) database, using the search strategy: nursing "and" urgency "and" emergency "and" sizing, four articles were selected for the present study. The data collection took place in March 2019. From the analysis of the productions, two thematic categories were constituted: "Profile of Nursing in Emergency Room, professional immaturity and inexperience: a reality in nursing care" and "Peculiarity of a Emergency Room in Nursing Dimensioning". The studies pointed out that nursing dimensioning is one of the weaknesses of hospital management and deserves an emphasis on the part of managers, in order to avoid overloading the team working in the sector and compromising nursing care for the patient. Nursing dimensioning is one of the most difficult and complex problems in hospital institutions, but it deserves special attention from managers since the PS unit offers variables that influence the dimensioning process.

Keywords: Nursing; Emergency Service, Hospital; Personnel Administration, Hospital.

\section{INTRODUÇÃO}

A falta ou a quantidade insuficiente do efetivo de profissionais de enfermagem em atividade nas instituições de saúde espalhadas pelo nosso país, ainda é um problema enfrentado pela classe. Para Matsushita, Adami e Carmagnani (2005), o dimensionamento dos profissionais de Enfermagem passou por três evoluções, começando em 1939 com o método intuitivo ou das relações de proporção e o método da variável de horas médias de cuidado. Em 1947 apareceria à introdução das variáveis relativas às ausências e em 1960 surge à introdução do Sistema de Classificação de Pacientes. O dimensionamento de profissionais de enfermagem é o processo inicial do método utilizado para alocar determinado número de profissionais que tem como objetivo prever a quantidade de funcionários por categoria, necessária para atender as necessidades da assistência de enfermagem seja direta ou indiretamente prestadas a clientela. 
Em unidades assistenciais especiais como: pronto socorro, pronto atendimento e centro obstétrico, não é possível à aplicação do dimensionamento por meio do sistema de classificação de paciente e nem referência de horas de intervenção, são utilizados o sitio funcional e o espelho semanal padrão. 0 primeiro consiste num parâmetro baseado em conhecimento profissional que considera as atividades desenvolvidas, a área e a carga semanal do local. Já o segundo é uma montagem gráfica e a distribuição das áreas operacionais com dias da semana, turno de trabalho e a categoria profissional (COFEN, 2017).

Os recursos humanos na área da saúde ainda não são rotulados como fator principal para o desenvolvimento do serviço e sim apenas uma parte do processo. Não recebem uma atenção exclusiva dos responsáveis pelo planejamento e desenvolvimento da assistência de enfermagem, causando um empecilho na distribuição adequada de profissionais de enfermagem, gerando uma assistência de baixa qualidade, esgotamento físico e mental dos profissionais relacionados no processo, levando-os assim a insatisfação ao exercer sua profissão. Porém o dimensionamento do pessoal de enfermagem acaba se tornando um desafio para os gestores do serviço em inovar e criar novas ideias para suprir as necessidades dessa classe (NICOLA, 2004).

Desse modo, a caracterização e avaliação dos níveis de cuidado dentro das instituições hospitalares, implicam na avaliação das peculiaridades de cada setor das instituições, contribuindo para o regulamento ideal da carga de trabalho e a distribuição adequada dos profissionais de saúde nelas atuantes (NICOLA, 2004).

Frente a este assunto, a questão de revisão do estudo foi: o que se tem publicado na literatura nacional acerca do dimensionamento de pessoal de enfermagem no Pronto Socorro? E objetivou-se identificar o que se tem publicado na literatura nacional acerca do dimensionamento de pessoal de enfermagem no PS.

\section{METODOLOGIA}


O presente estudo trata-se de revisão bibliográfica do tipo narrativa. A pesquisa narrativa, segundo Minayo (2013), caracteriza-se por uma pergunta ampla com intuito de identificar produção da temática. Desta forma, no estudo narrativo são consideradas representações ou interpretações do mundo e, portanto, não são abertas a comprovação e não podem ser julgadas como verdadeiras ou falsas, pois expressam a verdade de um ponto de vista em determinado tempo, espaço e contexto sócio histórico.

A pesquisa foi realizada na Biblioteca Virtual em Saúde (BVS), nas bases de dados Literatura Latino Americana e do Caribe em Ciências da saúde (LILACS), a partir da estratégia de busca: enfermagem "and" urgência "and" emergência "and" dimensionamento. O levantamento de dados ocorreu no período de março de 2019.

Os critérios de inclusão utilizados foram: textos completos, artigos publicados no idioma português, com disponibilidade na íntegra em suporte eletrônico e de forma gratuita e com recorte temporal dos últimos dez anos, tendo em vista escassez de publicações mais recentes, a fim de trazer dados atuais para a temática e que possam responder à questão de pesquisa.

Os critérios empregados para a exclusão dos artigos foram: não dispor de resumos, artigos que não estejam relacionados com o tema do presente estudo.

A técnica utilizada foi à análise de conteúdo de Minayo (2013). Este método, diz respeito às técnicas de pesquisa que permitem tornar replicáveis e válidas sobre dados de um determinado contexto, por meio de procedimentos. A análise temática, divide-se em três etapas que foram utilizadas para a realização deste trabalho. A primeira etapa é composta pela pré-análise onde foram utilizados os descritores no portal da Biblioteca Virtual em Saúde - BVS, conseguiu-se em um primeiro momento um total de 27 documentos, sendo 21 artigos, cinco teses e uma monografia.

Ao utilizar-se os filtros "texto completo disponível", "idioma" e "ano de publicação" e "Base de Dados", foram encontrados quatro estudos. A segunda etapa foi realizada a leitura exploratória dos artigos selecionados para ver se os mesmos se identificam com o objetivo proposto para este estudo. Após, foi realizada uma leitura seletiva onde os quatro artigos foram selecionados para 0 
presente estudo. A terceira etapa caracterizada pela análise e discussão dos resultados obtidos através das etapas anteriores e relativas à temática do estudo.

\section{RESULTADOS E DISCUSSÂO}

A partir da análise das produções, foram constituídas duas categorias temáticas: "Perfil da Enfermagem em Pronto Socorro, imaturidade profissional e inexperiência: uma realidade na assistência de enfermagem" e "Peculiaridade de um Pronto Socorro no Dimensionamento de Enfermagem". A fim de contribuir com uma melhor explanação dos resultados encontrados, realizou-se um quadro de extração de dados para auxiliar na análise, composto por: numeração, título, ano, objetivo e resultados (Tabela 1).

Tabela 1 - Caracterização dos estudos, 2019.

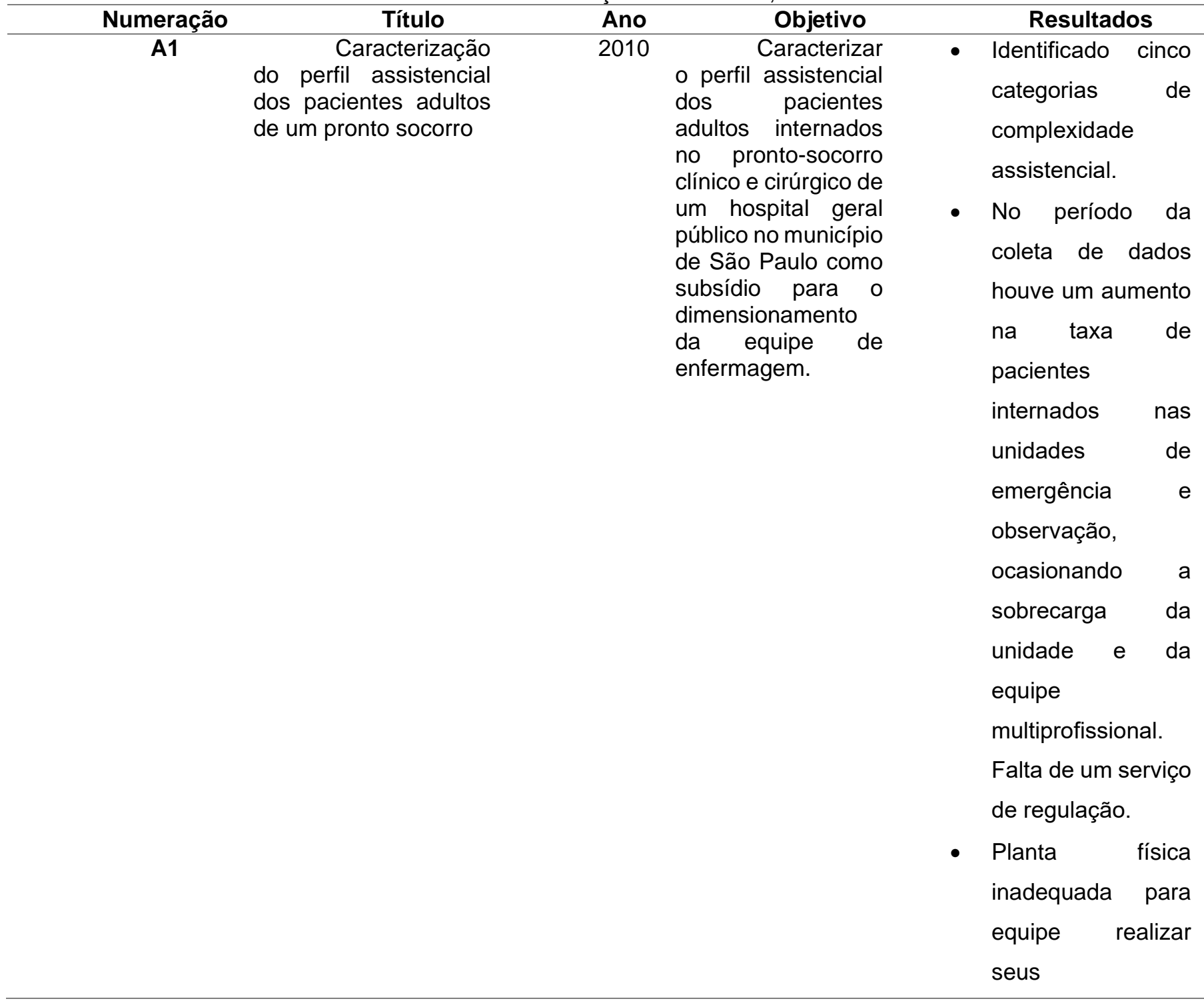


procedimentos,

causando desgaste

da

equipe,

comprovando

assim que a

caracterização dos

pacientes fornece

subsídios para o

dimensionamento

de enfermagem.

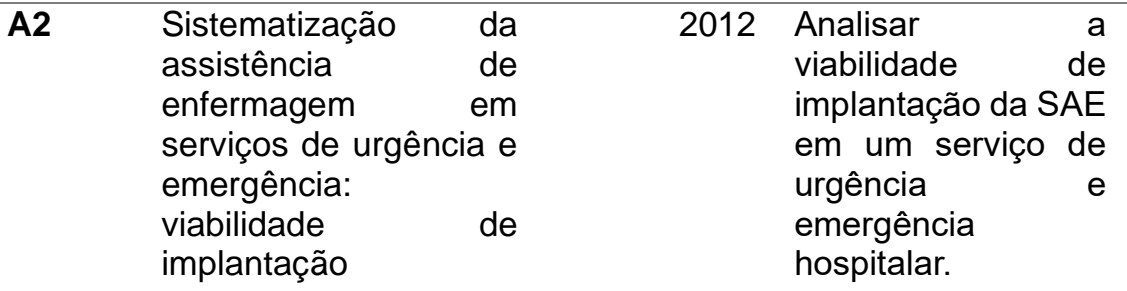

- Caracterização das competências da

equipe de

enfermagem desenvolvidas na instituição segundo a percepção de técnicos e auxiliares.

- A caracterização das competências da equipe de enfermagem desenvolvidas na instituição segundo a percepção dos enfermeiros.

Percepções dos enfermeiros sobre a SAE e sua implantação na instituição estudada.

> Dimensionamento torna-se inadequado perante o alto fluxo de pacientes e a estrutura física inadequada. 


\section{A3}

$\begin{array}{lr}\text { Indicativos para } & \text { o } \\ \text { dimensionamento } & \text { de } \\ \text { pessoal } & \text { de } \\ \text { enfermagem } & \text { em } \\ \text { emergência } & \end{array}$

2013 indicativos para dimensionamento de pessoal de enfermagem em serviços emergência.
- Processo de trabalho.

- Dimensionamento de pessoal de enfermagem.

- Indicativos para o dimensionamento de pessoal de enfermagem em emergência.

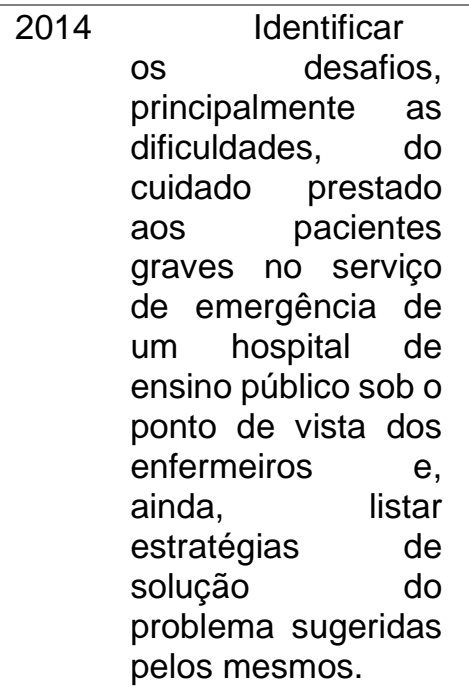

intensivos em um serviço hospitalar de emergência: desafios para os enfermeiros
- Dificuldades em relação ao déficit de recursos materiais, humanos e físicos frente à grande demanda de pacientes graves.

- Interferência na rotina do setor com a presença do paciente crítico foi em relação ao dimensionamento de pessoal.

- Estratégias sugeridas foram: aumentar os leitos de UTI, contratar funcionários, realizar treinamentos com a equipe e agrupar os pacientes graves. 
4 PERFIL DA ENFERMAGEM EM PRONTO SOCORRO, IMATURIDADE PROFISSIONAL E INEXPERIÊNCIA: UMA REALIDADE NA ASSISTÊNCIA DE ENFERMAGEM

Para um estudo realizado em uma unidade de PS, a equipe de enfermagem deve estar preparada, a fim de demonstrar agilidade, habilidade e um atendimento cauteloso e seguro para o paciente, lembrando que mesmo num atendimento de urgência o cuidado é o elo entre o paciente e o profissional. Para o líder enfermeiro trabalhar com situações de urgência e emergência requerem conhecimento técnico, cientifico e habilidades para liderar e trabalhar habilidades de comunicação, relacionamento interpessoal, tomada de decisão e competência clinica sempre pensando nas reais necessidades dos pacientes (MARIA; QUADROS; GRASSI, 2012).

Foram levantadas questões sobre a importância da instituição em incentivar a educação continuada, aprimorando 0 conhecimento de procedimentos, de condutas ajudando no crescimento da equipe, a fim de tornála prudente, treinada e especializada em atendimentos de urgência e emergência (MARIA; QUADROS; GRASSI, 2012). Isso reforça que nem todas as instituições seguem a portaria $N^{\circ} 3.194$, de 28 de novembro de 2017 que dispõe sobre 0 Programa para o Fortalecimento das Práticas de Educação Permanente em Saúde que tem como objetivo estimular, acompanhar e fortalecer a qualificação profissional dos trabalhadores da área da saúde para a transformação das práticas de saúde (BRASIL, 2017).

Ainda, percebe-se no relato dos enfermeiros que a equipe de enfermagem é muito inexperiente e com um nível de maturidade muito baixo, que prejudica a assistência de enfermagem necessitando de treinamento e capacitação técnica, causando-Ihes sentimentos de descontentamento e desapontamento (MARIA; QUADROS; GRASSI, 2012).

Para Felipe e Cardoso (2013), que realizaram um estudo em treze unidades de internação no Hospital das Clinicas de São Paulo relacionado ao conhecimento da equipe de enfermagem frente à situação do paciente apresentar uma parada cardiorrespiratória, a maioria dos profissionais relatou nunca ter trabalhado em unidades de atendimento a pacientes graves mesmo tendo em sua experiência profissional em torno de seis anos de atuação na área de enfermagem. Quando 
questionados sobre a atualização em parada cardiorrespiratória a maioria tinha realizado curso a mais de um ano e a outra parte nem se quer lembrava da última vez que tinha realizado o curso.

Outro ponto importante, que interfere na qualidade do profissional, é que existia uma baixa disponibilidade do mesmo estar aberto a receber atualizações e informações sobre o manejo com paciente crítico. Um ponto curioso do estudo que estava relacionado à participação dos profissionais durante uma parada cardiorrespiratória, cerca de $87 \%$ tinha participado de 0 a 2 vezes e $13 \%$ de 3 a 5 vezes. Ainda é grande o número de profissionais despreparados para o atendimento ao paciente grave. O despreparo pode mudar o prognóstico do paciente, ressaltam ainda, que quanto maior o conhecimento/experiência do trabalhador, maior é a eficácia do trabalho por ele realizado (FELIPE; CARDOSO, 2013).

Em um estudo que entrevistou enfermeiros de uma unidade de serviço hospitalar de emergência do hospital-escola do Paraná, constatou que principal alteração relatada pelos enfermeiros foi em relação ao dimensionamento de pessoal, presente em $60 \%$ das respostas. O quantitativo fixo de funcionários, dificulta o atendimento, pois o número de pacientes é oscilante nesta unidade, influenciando diretamente nos resultados da assistência prestada (ZANDOMENIGHI et al., 2014).

Em uma pesquisa realizada no serviço de emergência do Hospital de Cascavel, os profissionais relataram que durante a formação acadêmica a parte de gestão não ofereceu base para aplicar condutas perante as dificuldades enfrentadas como ditas por eles mesmos como, a falta de recursos humanos e a alta demanda de pacientes, que geram estresse e discussões entre a equipe de enfermagem (ZAMBIAZIE; COSTA, 2013).

O fato do dimensionamento não ser realizado de forma correta se observa nas falas dos entrevistados que relatam queda na qualidade do atendimento perante a falta de profissionais e a alta demanda de serviço, falta de medicação, baixa competência dos profissionais atuantes e o não incentivo da educação continuada por parte da instituição. Neste contexto os mais prejudicados por esses agravos são os pacientes que não receberão uma assistência de 
enfermagem adequada e segura e o trabalhador que adoece com a sobrecarga de trabalho (ZANDOMENIGHI et al., 2014).

\section{PECULIARIDADE DE UM PRONTO SOCORRO NO DIMENSIONAMENTO DE ENFERMAGEM}

Uma pesquisa no Hospital Geral de SP no setor de emergência, foram realizadas mais de 1.000 avaliações nos pacientes onde surgiram cinco categorias de complexidade assistencial sendo que cerca de 44,38\% se caracterizou na complexidade mínima, 22,15\% na complexidade intermediaria e 19,95\% na alta dependência. Mesmo apresentando pontuações diferentes, alguns estavam no limite de pontos entre fazer parte de uma categoria superior e inferior, devido os pacientes de PS serem muito instáveis (OHARA; MELO; LAUS, 2010).

A partir desses dados coletado verificou-se que no período da pesquisa um número elevado de internações na unidade do que a média do mês, sendo que a maioria não necessitava de internação e buscava consultas de rotina. Essa alta taxa de consultas sem internação acaba por sobrecarregar a unidade lotando os leitos oficiais, forçando a abertura de extras como macas espalhadas pelos corredores, reduzindo a planta física do setor, colocando os pacientes em condições inadequadas e a equipe em atuar em local inapropriado para realização de suas técnicas específicas (OHARA; MELO; LAUS, 2010).

Para Girardi et al. (2018), o dimensionamento de enfermagem é uma questão antiga entre a classe, sendo que para prestar uma assistência de qualidade precisa-se um quantitativo e qualitativo do pessoal de enfermagem condizente com a demanda do setor. O número inadequado da equipe implica de maneira importante na qualidade da assistência, visto que isto acarretara em sobrecarga de trabalho da equipe, gerando situações de estresses e cansaço, induzindo aos erros e eventos que causam malefícios aos pacientes, expondo tanto a equipe quanto a instituição a processos ético-legais.

O estudo realizado por Girardi et al. (2018), no setor de internação do PS de um hospital no Paraná, evidenciou 45,8\% $(n=1056)$ de pacientes que necessitam de cuidados intermediários, 35,6\% ( $n=821)$ de cuidados mínimos, $12,9 \%(n=299)$ de cuidados de alta dependência, 4,8\% $(n=112)$ de cuidados semiintensivos e $0,6 \%(n=15)$ cuidado intensivo. Em contrapartida outro estudo em 
uma unidade de internação médico-cirúrgico de um hospital universitário identificou um maior percentual de pacientes que necessitam de cuidados intermediários (42\%), seguido pelos cuidados mínimos $(21,7 \%)$, alta dependência $(32,5 \%)$ e cuidados semi-intensivos (3,8\%) (MORAES; LINCH; SOUZA, 2012)

O número de pacientes de cuidados semi-intensivos e intensivos nas unidades de internação é reduzido, devida as mesmas não ser destinadas a atender este tipo de paciente, pois demanda mais recursos tecnológicos, materiais e humanos. Já no PS encontram-se vários pacientes em cuidados intermediários que também não pertenceriam ao setor, mas devido ao elevado número de atendimentos e a falta de vaga para internação, acabam ficando alojados na unidade de emergência.

Outra fragilidade é alta taxa de pacientes que se encaixam na complexidade mínima, que na teoria demandam menos horas de enfermagem por dia, mas visto estarem em maiores números internados no PS demandam mais medicações, mais cuidados refletindo num maior tempo de enfermagem. O déficit de profissionais de enfermagem do PS evidenciado anteriormente, causando a sobrecarga de trabalho e posteriormente o absenteísmo, prejudicando a assistência de enfermagem ao paciente e colocando em risco a segurança do mesmo.

Quanto melhor dimensionado, mais eficaz será o cuidado com o paciente, pois se a equipe dispuser de um número menor de funcionários esse cuidado será prejudicado comprometendo a segurança do mesmo. No Brasil, o quantitativo de profissionais de enfermagem está bem abaixo se comparado aos demais países, pois não se tem dados que garantem um trabalho de enfermagem seguro, porém existe uma preocupação por parte da equipe com a segurança do paciente (SCHMOELLER; GELBCKE, 2013).

Há uma dificuldade em prever o quantiqualitativo da equipe de enfermagem em emergência adulto, principalmente por falta de uma regra, cujo fluxo de usuários é constante, além da variedade e simultaneidade de atividades e situações de riscos de morte encontrados no serviço, pois o PS pode apresentar o mesmo número de leitos de uma unidade, mas o tipo de cuidado de paciente de emergência é diferente e instável, estando ai o grande problema em dimensionar 
corretamente o pessoal de enfermagem do PS (SCHMOELLER; GELBCKE, 2013).

Ladeia et al. (2018), apontam para a importância da realização efetiva do dimensionamento do pessoal de enfermagem, assim como a dificuldade em ajustar o quantitativo adequado para que se possa realizar o trabalho com qualidade frente à demanda atendida. Há uma deficiência do pessoal de enfermagem do serviço de urgência e emergência, devido os mesmos trabalharem com um número reduzido de funcionários, atenderem pacientes com riscos iminente de morte e de alta complexidade, e servirem como unidades de internação para pacientes, que passam longos períodos internados, por isso, o dimensionamento será adequado quando houver a caracterização da clientela atendida no PS.

\section{CONLUSÃO}

Pode-se afirmar, diante disso, que os objetivos elencados foram alcançados, pois, foi possível refletir acerca do dimensionamento de enfermagem, principalmente voltado para os serviços de emergência, foi possível mostrar que o quadro de profissionais de enfermagem no PS merece uma atenção especial por parte dos gestores hospitalares e que o dimensionamento de enfermagem é um dos problemas mais complexos nas instituições hospitalares.

Torna-se crucial reconhecer a necessidade de os profissionais envolvidos nesse sistema de gerência reconhecer que a unidade de PS oferece variáveis que influenciam no processo de dimensionamento, como o espaço físico da unidade, a complexidade assistencial, instabilidade dos pacientes e a alta demanda de atendimentos. Isso reforça que a caracterização do paciente de PS serve como subsídios para um dimensionamento adequado.

É importante compreender que o serviço de emergência é complexo, isto se deve ao perfil dos pacientes. Acredita-se que as situações descritas sejam a realidade de muitas instituições do país e que este estudo possa ser um estímulo para que a categoria desenvolva métodos adequados para o dimensionamento de pessoal vislumbrando uma assistência com qualidade. 


\section{REFERÊNCIAS}

BRASIL. Ministério da Saúde. Portaria N 3.194, de 28 de novembro de 2017: Programa para o Fortalecimento das Práticas de Educação Permanente em Saúde. Brasília, 2017.2 Disponível em: <http://bvsms.saude.gov.br/bvs/saudelegis/gm/2017/prt3194_30_11_2017.html $\geq$

CONSELHO FEDERAL DE ENFERMAGEM. Resolução no 543/2017. Atualiza e estabelece parâmetros para o Dimensionamento do Quadro de Profissionais de Enfermagem nos serviços/locais em que são realizadas atividades de enfermagem. In: Conselho Federal de Enfermagem. Disponível em: $<$ http://www.cofen.gov.br/wpcontent/uploads/2017/05/Resolu\%C3\%A7\%C3\%A3o-543-2017-ANEXO-I.pdf> Acesso em: 28 de maio de 2019.

FELIPE, M. C.; CARDOSO, A. L. Conhecimento da equipe de enfermagem no atendimento a pacientes em parada cardiorrespiratória. Revista UNINGÁ. n. 37, p. 47-58, 2013. Disponível em: $<$ https://www.mastereditora.com.br/periodico/20131122_180254.pdf> Acesso em: 20 de maio de 2019.

GIRARD, C.; FELDHAUS, C.; OLIVEIRA, J. L. C.; SCHRAN, L. S.; LUZ, M. P.; TONINI, N. S.; BORDIN, V. Dimensionamento de pessoal de enfermagem em pronto socorro hospitalar. Revista de Administração em Saúde. v. 18, n. 71, 2018. Disponível em: <https://cqh.org.br/ojs-2.4.8/index.php/ras/article/view/95/139> Acesso em: 15 de maio de 2019.

LADEIA, L. F. A.; OLIVEIRA, L. B.; RIBEIRO, B. S.; CUNHA, F. O.; TEÓFILO, V. A.; RAMOS, L. G. D.; ANDRADE, J. M. O.; ALMEIDA, E. W. S.; MAGALHÃES, D. O. L.; ANDRADE, F. M. Dimensionamento da equipe de enfermagem em um pronto-socorro de um hospital escola. Revista Eletrônica Acervo Saúde. n. 13, 2018.

MARIA, M. A.; QUADROS, F. A. A.; GRASSI, M. F. O. Sistematização da assistência de enfermagem em serviços de urgência e emergência: viabilidade de implantação. Revista Brasileira de Enfermagem. v. 65, n. 2, 2012. DOI: https://doi.org/10.1590/S0034-71672012000200015

MATSUSHITA, M. S.; ADAMI, N. P.; CARMAGNANI, M. I. S. Nursing staff dimensioning in the hospitalization units of the Hospital São Paulo. Acta Paulista de Enfermagem. v. 18, n. 1, p. 9-19, 2005. DOI: https://doi.org/10.1590/S010321002005000100002

MINAYO, Maria Cecília Souza. O desafio de conhecimento: pesquisa qualitativa em saúde. São Paulo: Hucitec, 2013.

NICOLA, A. L. Dimensionamento de Pessoal de Enfermagem no Hospital Universitário do Oeste do Parana. Ribeirão Preto. 153f. Tese de Doutorado-Escola de Enfermagem da Universidade de São Paulo,2004. 
OHARA, R.; MELO, M. R. A. C., LAUS, A. M. Caracterização do perfil assistencial dos pacientes adultos de um pronto socorro. Revista Brasileira de Enfermagem. v. 63, n. 5 , p. $749-54,2010$. DOI: https://doi.org/10.1590/S003471672010000500009

SCHMOELLER, R.; GELBCKE, F. L. Indicators for the measurement of emergency nursing personnel. Text Context Nursing. v. 22, n. 4, p. 971-9, 2013. DOI: https://doi.org/10.1590/S0104-07072013000400013

ZAMBIAZI, B. R. B.; COSTA, A. M. Gerenciamento de enfermagem em unidade de emergência: dificuldades e desafios. Revista de Administração em Saúde. v. 15, n. 61, 2013.

ZANDOMENIGHI, R. C.; MOURO, D. L.; OLIVEIRA, C. A.; MARTINS, E. A. P. Intensive care in hospital emergency services: challenges for nurses. Revista Mineira de Enfermagem. v. 18, n. 2, p. 404-14, 2014. DOI: https://doi.org/10.5935/1415-2762.20140031 